\title{
The NASA/JPL program in microgravity fundamental physics
}

\author{
U. E. Israelsson \\ Jet Propulsion Laboratory, California Institute of Technology, Mailstop 79-5, \\ 4800 Oak Grove Drive, Pasadena, CA 91109, USA \\ E-mail: ulf@squid.jpl.nasa.gov \\ M. C. Lee \\ National Aeronautics and Space Administration, Microgravity Research Division, \\ Code UG, Washington, DC 20546-0001, USA \\ E-mail: mark.lee@hq.nasa.gov
}

\begin{abstract}
The National Aeronautics and Space Administration (NASA) has been supporting research in microgravity low temperature physics for about 20 years. In the last 10 years, the program has seen significant growth in the number of funded investigations and in the breadth of the research activities being pursued. Currently, flight experiments are being performed exclusively on the Space Shuttle. For the future, a cryogenic Space Station facility is being developed by the Jet Propulsion Laboratory and industrial partners to support the microgravity needs of the international scientific community. The facility will be attached to the Japanese Experiments Module's Exposed Facility and will operate with a 6 month helium cryogen lifetime. Flights of the facility are planned at 2 year intervals starting in 2003 with each flight accommodating multiple scientific experiments. Capabilities, conceptual designs and development plans for the facility are discussed along with a summary of potential near term flight candidate experiments.
\end{abstract}

PACS: $67.90 .+z, 04.80 .-y$

\section{Introduction}

There are three different organizations within NASA that supports scientific research. Code Y supports research in Earth science, Code S supports research in planetary exploration and astronomy, and Code U supports research in life and microgravity science and applications. The Microgravity Research Division is part of Code U and sponsors research in the areas of biotechnology, combustion science, fluid physics, fundamental physics, and material science. The fundamental physics program is managed for NASA by the Jet Propulsion Laboratory (JPL) and aims at studying far-reaching physics questions that are obscured by gravity on the Earth. To accomplish the program goals, NASA funds a large number of ground investigators from which to select the best future flight experiments. Funds are also being expended on development of advanced technology to support the needs of future flight experiments.

In the early eighties the fundamental physics program consisted of a small community of investi- gators supported by NASA's Physics And Chemistry Experiments Program (PACE). This community expanded in the late eighties and early nineties to involve many major Universities. Currently NASA is funding 30 investigators (6 for potential flight) in the fundamental physics area. 26 of the investigations are in the low temperature and condensed matter physics area, 4 investigations are in the laser cooling and atomic physics area, and 2 investigations are in the relativity and gravitational physics area. Historically, the program has focused on research in the condensed matter physics area, primarily on critical point studies, although significant growth has occurred recently in the other research areas.

\section{Shuttle program history}

In 1985 the Superfluid Helium Experiment [1], developed by the Jet Propulsion Laboratory, demonstrated the containment and control of liquid helium aboard the space shuttle and the feasibility of supporting a science instrument insert within a 
liquid helium dewar. Containment using the fountain pressure was accomplished by pumping with space vacuum on the helium through a porous plug. The porous plug and vent plumbing must be precisely adapted to the expected operating conditions for containment to occur.

In 1992 the Lambda-Point Experiment (LPE)[2], developed by Stanford University, JPL and Ball Aerospace, added nanokelvin high resolution thermometry to this capability which allowed a precise test of the Nobel Prize winning Renormalization Group (RG) theory of critical phenomena [3] to be performed. The LPE demonstrated that the superfluid transition in ${ }^{4} \mathrm{He}$ is sharp to within about one nanokelvin, or about two orders of magnitude closer to the critical point than accessible in a ground experiment. The heat capacity exponent measured by LPE $(\alpha=-0.0128 \pm 0.0004)$ agrees with RG predictions [4], but is about fifteen times more accurate than the best theoretical calculations. By making use of ground-based measurements of the correlation length exponent, $v$ [5], LPE was able to demonstrate to a high precision the validity of the hyperscaling relation $d v=2-\alpha[6]$, where $d=3$ represents the dimensionality of the system. This scaling relation is one of the cornerstones of the theory giving confidence that the theory of critical phenomena is in effect an exact description of continuous phase transitions. Further, by making use of thermal transient data in the normal phase LPE was able to extend ground observations of thermal conductivity closer in to the transition. The data is consistent with ground data [7] further from the transition and with calculations based on dynamic renormalization group theory techniques [8]. Perhaps equally significant, LPE demonstrated that advanced technology, high resolution experiments can be made to survive the shock associated with launch and can operate flawlessly in the hostile space environment. Subsequent flight projects, including the plans for experimentation aboard the space station relies heavily on the pioneering work developed for the LPE flight.

In 1997, the Confined Helium Experiment $(\mathrm{CHeX})$ will use the unique properties of liquid helium to perform a high resolution test of the theory of finite size effects [9]. CHeX will investigate the shape of the heat capacity curve very near the lambda transition of a sample confined in one of the dimensions to about 50 microns thickness. Confinement is accomplished by stacking nearly 4004 $\mathrm{cm}$ diameter 50 micron thick Silicon wafers on top of each other with a 50 micron gap space between.
The last of the Shuttle based experiments, Critical Dynamics in Microgravity (DYNAMX) is tentatively scheduled to fly in 2001. DYNAMX will study the rich, largely unexplored, properties of superfluid helium driven away from equilibrium by introduction of a heat current [10]. Thermal conductivity data in the non-linear region will be collected as well as heat capacity data just below the superfluid transition. Heat currents below about $100 \mathrm{nW} / \mathrm{cm}^{2}$ will be used predominantly.

While necessary and productive, shuttle based experiments are short term, lasting no longer than ten days; expensive; and require up to 7 years for development. It is clear that future experiments will require longer duration experiment times than possible on the shuttle. Therefore, the National Research Council's Space Studies Board and other advisory groups have recommended that NASA develop a cryogenic capability on the Space Station[11] for use by the fundamental physics community.

\section{Space station facility description}

The facility will be implemented by a science, Industry and JPL partnership of joint participation through all phases of definition, development and test. JPL will be responsible for development of the instruments and management of the overall activity. The Industry partner, Ball Aerospace and Technology Corporation (BATC) was selected through a competitive technical selection process. BATC will be responsible for development of the facility flight hardware and the ground data system for controlling the experiments.

A conceptual design for the facility that meets all the imposed requirements has already been developed. An artist's rendering of the facility is shown in Fig. 1. The design provides 6 months of cryogenic lifetime operating as an attached payload to the Japanese Experiment Module's Exposed Facility. The facility carries about two hundred and sixty liters of helium and accommodates two instrument inserts, one from either end of the cylindrical facility. Each instrument must fit within a volume defined by a cylinder with a diameter of $20 \mathrm{~cm}$ and a length of $50 \mathrm{~cm}$. Other assumed constraints for each instrument is a mass limitation of $50 \mathrm{~kg}$, a wire and plumbing count similar to LPE, and a power dissipation at helium temperatures of $10 \mathrm{~mW}$. In addition to the necessary readouts for the helium dewar, the flight electronics will also accommodate all cards necessary to operate the two instruments. The instrument portion of the electronics provides germanium thermometry readouts and heater con- 
trol circuits, 8 SQUID controllers, 4 precision heater drivers, and also ten extra slots for electronics cards beyond the capability of the LPE instrument. Passive vibration isolation of the facility is provide directly from the Space Station structure. The resonant frequency of the isolator is about $3 \mathrm{~Hz}$, providing about a factor of 10 attenuation at a frequency of $10 \mathrm{~Hz}$. To provide experimenters with a direct correlation of important environmental influences to their science data, the facility provides the output from a 3 -axis accelerometer and a charged particle monitor. The 3 -axis accelerometer is sampled at $250 \mathrm{~Hz}$ and will provide accurate g-jitter information up to $100 \mathrm{~Hz}$ at levels down to a few $\mu \mathrm{g}$. The charged particle monitor will have a sampling rate of up to $100 \mathrm{~Hz}$ and a sensitivity of about $2 \mathrm{MeV}$ for protons and heavier particles.

After the two instruments have been constructed and tested, they will be integrated with the Space
Station facility at JPL. Following integration and cool down to helium temperatures, the facility and instruments will go through system testing and environmental sensitivity testing at JPL. The integrated system will then be shipped cold to Kennedy Space Center for integration with the Shuttle orbiter. The dewar is last serviced on the launch pad prior to payload bay door closure launch activities. Transfer from the Orbiter to the Space Station is accomplished by crew internal vehicle activity using Orbiter and Space Station remote manipulator systems.

Turn-on and check-out will be performed at the Payload Operations Control Center (POCC) for the Space Station and routine operations will be conducted from individual remote POCCs located at investigator facilities using telemetry and command services from the Space Station POCC. The facility will operate continuously for 6 months or

Fig. 1. An artist's rendering of the planned Space Station facility for performing fundamental physics experiments. The Payload Interface Unit (PIU) serves as the mechanical interface to the Space Station. The PIU is attached to the facility through passive vibration isolators. The trunnion pins are used to secure the facility in the Shuttle cargo-bay. Transfer from the Space Shuttle to the Space Station is accomplished with the grapple fixture and robotic arms. 
until the cryogen is expended and then wait passively for the next opportunity to transport back to Earth.

During the 6 months operation of one flight, a second pair of instruments will be developed, integrated and tested with another flight dewar in preparation for the next flight. Upon return to Earth, the facility will proceed through a brief checkout and the instruments and dewar from the first flight will be removed and replaced with the flight ready instruments and dewar for the next flight. This exchange of instruments and dewars will allow re-flight as often as every $20-24$ months.

\section{Science selections}

Science participants will be determined through the NASA Research Announcement (NRA) peer review process. Two potential flight experiments for the Space Station facility have recently been selected [14]. The Microgravity Scaling Theory Experiment (MISTI) [15] will perform very precise measurements of thermodynamic properties near the liquid/vapor critical point of $3 \mathrm{He}$. In particular, MISTI will measure the heat capacity exponent $(\alpha)$, the isothermal compressibility exponent $(\gamma)$, and the exponent characterizing the relation between pressure and density along the critical isotherm $(\delta)$. These exponents will be used for a self-consistent test of scaling law predictions.

The Superfluid Universality Experiment (SUE) [16] will measure the superfluid density exponent along the lambda line of helium in microgravity. These measurements along with measurements of the heat capacity exponent along the lambda line in the $0.01 \mathrm{~g}$ facility at JPL [17] will be used to check the universality prediction of the theory of critical phenomena.

Two additional flight definition investigations are targeted to be selected from a NRA solicitation currently under review. Subsequent NRAs will be issued at two year intervals for future experiment selections.

\section{Conclusions}

The microgravity fundamental physics program of NASA's Microgravity Research Division have experienced significant growth in the early 1990's. To meet the need for extended experimentation time in microgravity over the coming decades, NASA and JPL have teamed with Industry to develop a Microgravity Physics Facility for use on the International Space Station. The facility attaches outside the Space Station to the Japanese Experiment Module's Exposed Facility and provides a 6 month lifetime for operation of two instruments per flight. The international scientific community is invited to collaborate with U.S. researchers in developing science experiments for this facility via the NASA Research Announcement process. The first flight of the facility is targeted for the year 2003 .

\section{Acknowledgements}

This work was carried out by the Jet Propulsion Laboratory, California Institute of Technology under a contract with NASA. We acknowledge stimulating discussions with Rich Reinker and Reuben Ruiz.

1. P. V. Mason et. al, Proc. Intern. Cryogenic Engineering Conf. West Berlin, April 22-25, 1986, Butterworth Guilford, Surrey (1986).

2. J. A. Lipa, D. R. Swanson, J. A. Nissen, T. C. P. Chui, and U. E. Israelsson, Phys. Rev. Lett. 76, 944 (1996).

3. K. G. Wilson, Phys. Rev. B4, 3174 (1971).

4. J. C. Le Guillou and J. Zinn-Justin, Phys. Rev. B21, 3976 (1980); D. Z. Albert, Phys. Rev. B25, 4810 (1982).

5. L. S. Goldner, N. Mulders, and G. Ahlers, J. Low Temp. Phys. 93, 131 (1992); D. R. Swanson, T. C. P. Chui, and J. A. Lipa, Phys. Rev. B46, 9043 (1992).

6. B. D. Josephson, Proc. Phys. Soc. 92, 269 (1967).

7. W. Y. Tam and G. Ahlers, Phys. Rev. B32, 5932 (1985).

8. V. Dohm, Phys. Rev. B44, 2697 (1991); Z. Phys. B61, 193 (1985).

9. D. R. Swanson, J. A. Nissen, X. Qin, P. R. Williamson, J. A. Lipa, T. C. P. Chui, U. E. Israelsson, and F. M. Gasparini, J. of Spacecraft and Rockets 33, 154 (1996).

10. Principal investigator, R. V. Duncan, University of New Mexico.

11. Microgravity Research Opportunities for the 1990s, unpubl. report by the National Research Council's Space Studies Board.

12. J. A. Lipa, D. R. Swanson, and T. C. P. Chui, Cryogenics 34, 341 (1994).

13. X. Qin, J. A. Nissen, D. Swanson, P. R. Williamson, D. A. Stricker, J. A. Lipa, T. C. P. Chui, and U. E. Israelsson, Czech. J. Phys. 46, 2857 (1996).

14. NRA-94-OLMSA-05, November 29, 1994

15. Principal investigator, M. Barmatz, JPL

16. Principal investigator, J. Lipa, Stanford University.

17. M. Larson, F. Liu, and U. E. Israelsson, Czech. J. Phys. 46, 179 (1996). 